\title{
Cell-mediated immune response after vasectomy in rats
}

\author{
S. W. McDonald and J. Halliday \\ Department of Anatomy, University of Glasgow, Glasgow G12 8QQ, UK
}

\begin{abstract}
Summary. This study investigated the development of a cell-mediated immune response after vasectomy in Swiss Albino rats, by comparing the development of the thymusdependent lymphoid tissue of the regional testicular lymph node and the spleen in vasectomized and in sham-operated control animals. Frozen sections were used and thymus-dependent regions were stained by immunocytochemistry. After vasectomy, the areas occupied by the paracortex in the lymph node sections showed a significant increase in size; the thymus-dependent regions of the spleen, in contrast, showed no change. The regional lymph node, rather than the spleen, seems to be important in cellmediated and humoral immune responses to vasectomy.
\end{abstract}

Keywords: lymph node; spleen; cell-mediated immunity, T-areas; rat

\section{Introduction}

Humoral immunity to spermatozoan antigens after vasectomy is well documented in man and laboratory animals (Rumke \& Titus, 1970; Shulman et al., 1972; Samuel et al., 1975; Bigazzi et al., 1977). Morphological changes, consistent with this humoral response, have been described in the first regional testicular lymph node of rats after left unilateral vasectomy (McDonald \& Scothorne, 1986, 1989). Al-Saffar (1987) investigated the immune response to vasectomy in rat spleen by studying the areas occupied by the white pulp in spleen sections and the content of germinal centres; he concluded that there was no response.

Compared with humoral immunity, cell-mediated immunity to spermatozoa after vasectomy is poorly documented. This study used immunocytochemistry to stain thymus-dependent lymphoid tissue, the tissue involved in cell-mediated immune responses, in frozen histological sections of the regional testicular lymph node and of the spleen of rats after left unilateral vasectomy and of control sham-operated animals. By determining the sectional areas occupied by thymus-dependent lymphoid tissue, the study aimed to determine whether either organ is involved in a cell-mediated immune response to vasectomy.

\section{Materials and Methods}

\begin{abstract}
Animals
Young adult Swiss Albino rats from an inbred colony maintained in the Department of Anatomy, at the University of Glasgow, were used. Their ages at operation ranged from 81 to 88 days.

A total of 14 rats was used, seven vasectomized and seven sham-operated. Only five vasectomized and five shamoperated rats were used for renal node analysis; in these rats, the lymphatics of the left testis drained directly to the left renal node. In the remainder, the left testis drained to other para-aortic nodes or directly to the cisterna chyli. The spleens of all rats were used in the analysis.
\end{abstract}

\section{Surgical technique}

Left unilateral vasectomies were performed under pentobarbitone sodium anaesthesia. The ductus deferens was doubly ligated with silk close to the epididymis and the intervening portion was transected. By carrying out the 
vasectomy at this site, it was anticipated that the sperm granuloma, a chronic inflammatory lesion at the site of leakage of spermatozoa and the presumed site of access of spermatozoan antigens to the immune system, would form at the cauda of the epididymis from which lymph drains directly to the regional testicular node, as shown by McDonald and Scothorne (1988). The cremaster muscle and skin were closed with catgut and silk sutures, respectively. For a sham operation, two loose ligatures were passed around the ductus deferens, which was not transected. After operation, the animals were inspected regularly to ensure good healing and the absence of cryptorchidism. The rats were killed with pentobarbitone sodium from 13 to 15 weeks after operation.

\section{Collection of material}

In vasectomized and sham-operated rats, the scrotal cavities were opened and the testes and epididymides exposed. The position of the sperm granuloma was noted. The abdomen was also opened as far as the lower border of the rib-cage. The lymphatic drainage pattern of the left testis was demonstrated by injection of approximately $0 \cdot 1 \mathrm{ml}$ pontamine sky blue dye beneath the tunica albuginea. After injection, the site was gently massaged to encourage filling of the lymphatics, and kept moist using mammalian Ringer's solution. The drainage pattern was studied using a binocular microscope and recorded by a drawing. Those left renal nodes, as defined by Tilney (1971), that received lymph directly from the testis were dissected out, weighed and then frozen on a cryostat (Frigocut 2800) deep-freezing station. The spleen was then removed, weighed and divided transversely into six portions and each portion was frozen on dry ice. The left and right testes were also removed and weighed.

The mean weights of the left renal lymph nodes and of the spleens of vasectomized and control rats were compared by two-tailed Student's $t$ test.

\section{Histological preparations}

The renal nodes were cut serially at $7 \mu \mathrm{m}$ and four frozen sections mounted on each slide, dried for $1 \mathrm{~h}$ and fixed in acetone for $10 \mathrm{~min}$. They were then dried for a further $30 \mathrm{~min}$ and stored at $-20^{\circ} \mathrm{C}$. Random frozen sections of each portion of the spleens were prepared, mounted, fixed and stored as described for the lymph node sections.

The frozen sections of lymph node and spleen were stained to show thymus-dependent areas by the indirect immunoperoxidase reaction. The technique was a modification of that described by Van Noorden (1986); the second antibody was diluted with $10 \%$ rat serum in phosphate-buffered saline.

For immunocytochemistry, three primary antibodies, mouse monoclonal antibodies against different populations of rat lymphocytes, were used: W3/13 (all T-lymphocytes), OX12 (all B-lymphocytes) and OX8 (suppressor T cells and non-helper T cells; Seralab, Crawley Down, Sussex, UK). Pilot studies using W3/25, which binds to helper T cells, gave staining of macrophages and was, therefore, inappropriate for the study.

The sections stained using W3/13 were measured. The OX12- and OX8-reacted sections served as controls. The antibodies were derived from culture supernatant and used at a dilution of 1:3. In addition, sections treated with $1 \%$ bovine serum albumin in phosphate-buffered saline, instead of primary antibody, served as further controls.

The second antibody was a biotinylated horse anti-mouse immunoglobulin conjugated to peroxidase (dilution 1: 50, Vector Laboratories, Peterborough, Cambs, UK).

The slides were reacted with both antibodies and placed in diaminobenzidine tetrahydrochloride dihydrate (Aldrich Chemical Co., Gillingham, Kent, UK), which reacts with the peroxidase to give a brown colour at the site of antibody localization. They were then counterstained using haematoxylin, dehydrated in graded alcohols and mounted in Histomount (Hughes and Hughes Ltd, Romford, Essex, UK).

\section{Quantitative analysis}

A Wild binocular microscope with camera lucida attachment was used and the outline of each W3/13 section of lymph node was traced on to graph paper with squares of sides $2 \mathrm{~mm}$ long. The intersections of the lines of the graph paper could be seen superimposed on the image of the section. When the intersection of two lines lay on an immunocytochemically stained part of the cortex, it was marked by a cross; when an intersection was on a negatively stained area of cortex, it was marked by a dot (Fig. 1). This method of determining the areas of T-cell-dependent and B-celldependent cortex was adopted because the specifically stained areas tended to blend at their edges with surrounding cortex making it impossible to define a distinct boundary. The areas occupied by both positively and negatively stained regions of cortex formed an important quantitative control; as changes consistent with humoral immunity are well documented, any result indicating smaller areas of negatively stained cortex after vasectomy compared with sham operation would suggest inaccurate assessment. The magnification of each section traced was determined.

The numbers of crosses and dots observed were divided by the square of the magnification of the system to eliminate small variations in the magnifications between counting sessions. For each series of lymph node sections, the values obtained from the point counting analysis of $\mathrm{T}$-cell and B-cell regions of cortex were added. The means of the values obtained for the experimental and control material were compared by two-tailed Student's $t$ test.

The sections of spleen were measured in a different way. In the sections stained using the W3/13 antibody the thymus-dependent regions were sharply demarcated and easily distinguished from the surrounding negatively-stained white pulp. A Wild binocular microscope was used and the perimeter of each section stained using W3/13 antibody 


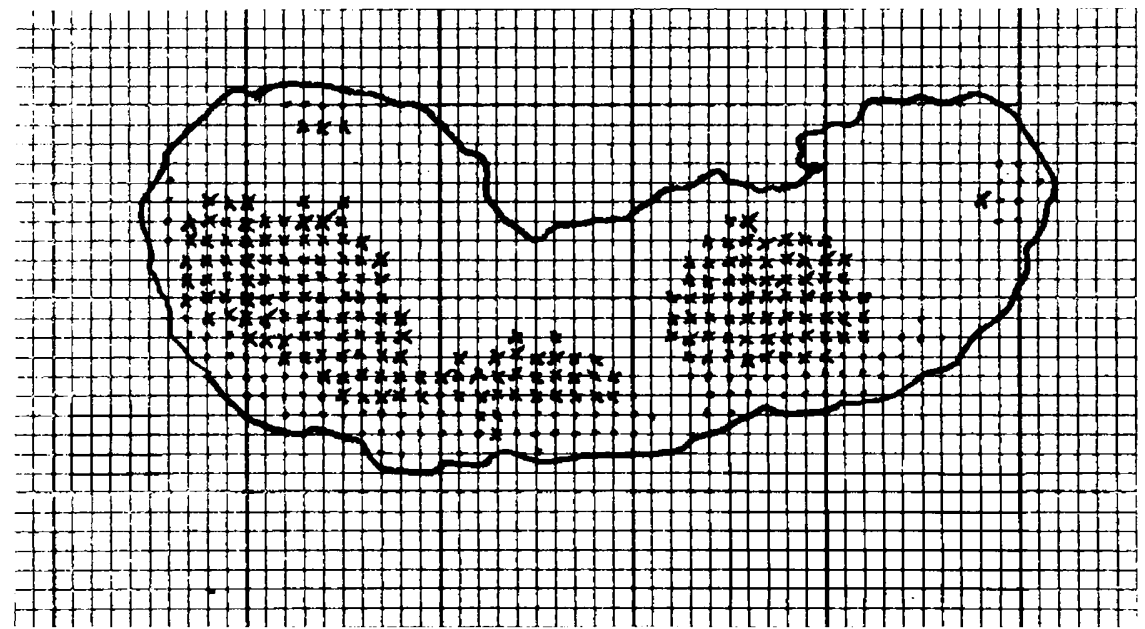

Fig. 1. Camera lucida drawing of a rat lymph node section (actual size), projected on to squared paper. Line intersections on which the image of $\mathrm{T}$-cell areas fell are marked by a cross, those on which B-cell areas fell are marked by a dot. For this section, the image of the thymusdependent areas fell on 198 line intersections, while the bursa-dependent cortex fell on 102 intersections.

was traced onto paper. The individual thymus-dependent regions were then traced onto the drawing. The total areas of the individual thymus-dependent regions were measured on an electronic planimeter (Kontron, Munich, Germany). The magnification of the system was recorded for each section, but no alteration in magnification occurred between drawings; there was, therefore, no need to correct the areas of the drawings representing thymus-dependent lymphoid tissue for variations in magnification.

The areas of the thymus-dependent lymphoid tissue for the six sections of each spleen were added. The means of the sums of the areas in vasectomized and sham-operated rats were compared by two-tailed Student's $t$ test.

\section{Results}

\section{Gross appearance}

The rats were healthy and ranged in weight from 345 to $438 \mathrm{~g}$. The testes of both sides were normal; none had degenerated. Moderate-sized granulomas were seen in or on the left cauda epididymidis of all vasectomized rats.

\section{Weights of lymph nodes and spleens}

The weights of the left renal nodes receiving testicular lymph in vasectomized rats ranged from 9.02 to $16.44 \mathrm{mg}$ and in sham-operated rats from 2.81 to $8.76 \mathrm{mg}$ (Table 1); there was a significant difference, $P<0 \cdot 01$.

The weights of the spleens of all the vasectomized rats ranged from 0.69 to $0.95 \mathrm{~g}$, whereas those of controls ranged from 0.70 to $0.80 \mathrm{~g}$ (Table 1 ); there was no significant difference between the two groups.

\section{Immunocytochemical staining}

The staining of the sections of the lymph node and spleen reacted with monoclonal antibodies W3/13, OX8 and OX12 and with bovine serum albumin only were compared. The sections reacted with bovine serum albumin only showed little staining. Sections reacted with W3/13 showed clear staining of the paracortex (Fig. 2a) and the periarterial lymphoid sheaths (Fig. 3a) of lymph node 
Table 1. Means and standard deviations of parameters assessed in an analysis of cell-mediated immunity after vasectomy in rats

\begin{tabular}{|c|c|c|c|}
\hline Parameter & Vasectomy & $\begin{array}{l}\text { Sham } \\
\text { operation }\end{array}$ & \\
\hline $\begin{array}{l}\text { Lymph node weight (mg) } \\
\text { Spleen weight }(\mathrm{g})\end{array}$ & $12 \cdot 60 \pm 3 \cdot 20$ & $6 \cdot 00 \pm 2.04$ & $P<0.01$ \\
\hline $\begin{array}{l}\text { Spleen weight }(\mathrm{g}) \\
\text { Values from point-counting analysis of }\end{array}$ & $0.76 \pm 0.09$ & $0.75 \pm 0.04$ & NS \\
\hline T-cell-areas of lymph node & $3.52 \pm 1.23$ & $1.74 \pm 0 \cdot 61$ & $P<0.05$ \\
\hline B-cell-areas of lymph node & $2 \cdot 21 \pm 0.65$ & $1 \cdot 18 \pm 0 \cdot 20$ & $P<0.01$ \\
\hline $\begin{array}{l}\text { Sum of areas representing T-cell regions in drawings of splenic } \\
\text { sections }\left(\mathrm{cm}^{2}\right)\end{array}$ & $18 \cdot 9 \pm 4 \cdot 1$ & $17 \cdot 1 \pm 1 \cdot 0$ & NS \\
\hline
\end{tabular}

NS: not significant

and spleen, respectively. In the nodes, there was also staining of the medullary cords, although it was somewhat lighter than in the paracortex. The outer cortex of lymph nodes and the regions of the splenic white pulp closest to the red pulp showed little reaction product, with only scattered positively-stained cells. With the OX8 antibody, stained lymphocytes were scattered through the cortex and medullary cords of the lymph nodes, but were principally located in the paracortex; in the splenic sections, their distribution was similar to that of the W3/13-positive cells. In the sections of the lymph nodes and of the spleens, however, the OX8-positive cells were too widely dispersed to allow meaningful measurements of T-cell-dependent areas. Sections reacted with antibody OX12 showed generalized nonspecific light-brown staining. Cells with strong positive staining were scattered in the node, but were found in large numbers in the outer cortex (Fig. 2b), whereas in sections of spleen they were principally located in the outer parts of the Malpighian bodies (Fig. 3b). The distribution of positive staining in the OX8- and OX12-reacted sections was consistent with the localization of the $\mathrm{W} 3 / 13$ reaction product.

\section{Measurement of thymus-dependent lymphoid tissue}

The point-counting analysis of $\mathrm{T}$-cell areas of cortex of lymph nodes gave values that ranged from 1.63 to 4.83 for vasectomized rats and 0.96 to 2.63 for controls (Table 1); there was a significant difference between the means at $P<0.05$. For B-cell areas of cortex the ranges were 1.09 to 2.72 and 0.96 to 1.41 for vasectomized and control rats, respectively (Table 1); the means showed a significant difference at $P<0.01$.

The sum of the areas of the camera lucida drawings representing thymus-dependent regions of the white pulp of the six sections of each spleen ranged from 13.5 to $26.0 \mathrm{~cm}^{2}$ in vasectomized rats and from 16.0 to $18.6 \mathrm{~cm}^{2}$ in sham-operated rats (Table 1); there was no significant difference between the means.

\section{Testicular weights}

The weights of the testes ranged from 1.34 to $1.62 \mathrm{~g}$ on the left side and from 1.25 to $1.51 \mathrm{~g}$ on the right in vasectomized rats. They ranged from 1.45 to $1.65 \mathrm{~g}$ on the left side and from 1.30 to $1.52 \mathrm{~g}$ on the right in sham-operated rats.

\section{Discussion}

Previous attempts to demonstrate cell-mediated immunity after vasectomy in man and laboratory animals have relied on in vitro assays. Because of technical difficulties, few such studies of cellmediated immunity have been performed and the results are difficult to compare (Brannen et al., 1974; Nagarkatti \& Rao, 1976; Tumboh-Oeri \& Roberts, 1979). In this study in rats, a new 
(a)

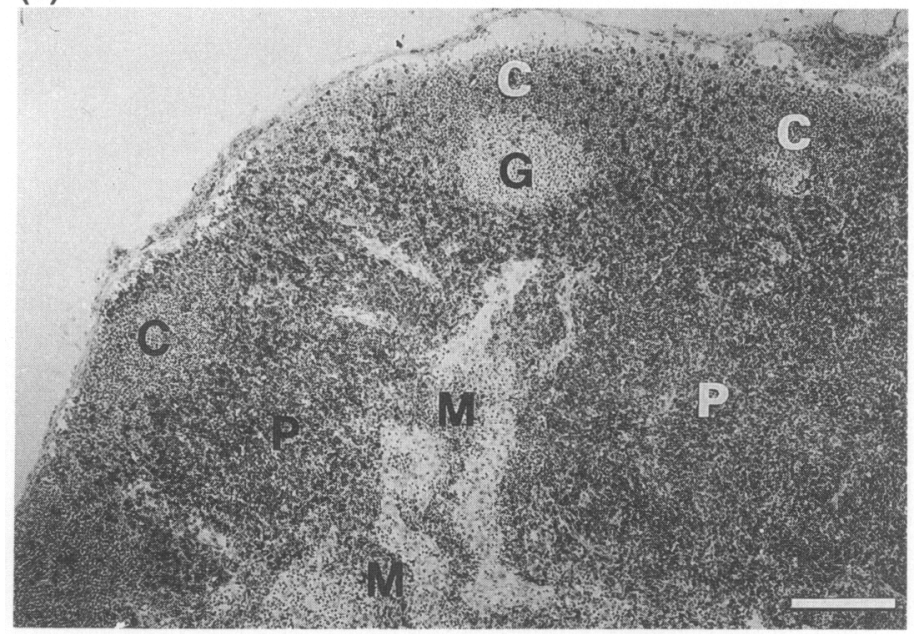

(b)

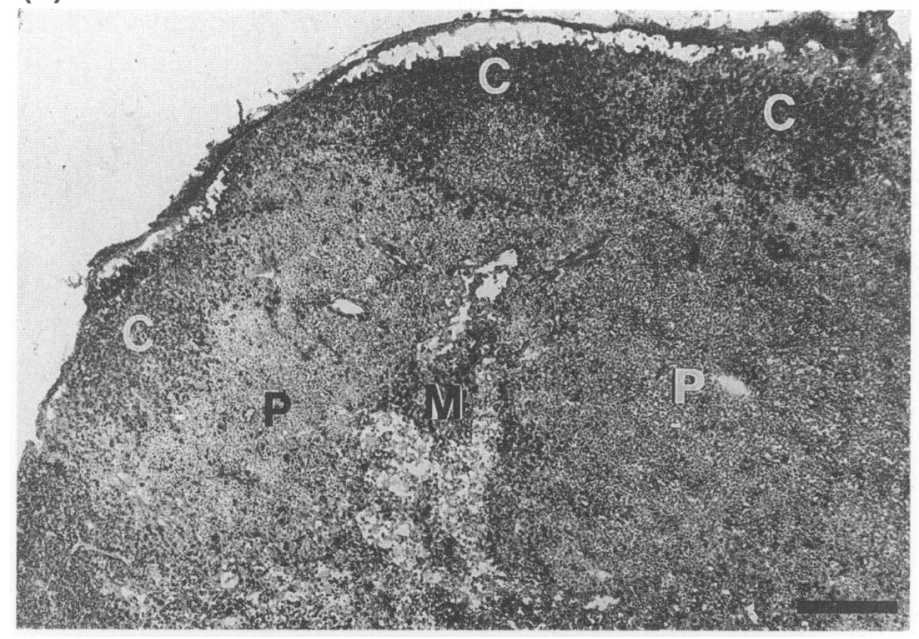

Fig. 2. (a) Section of lymph node from a vasectomized rat, showing paracortex (P) immunostained using monoclonal antibody W3/13. The outer cortex $(C)$ shows a germinal centre $(G)$. The medullary cords (M) are also visible. Bar: $200 \mu \mathrm{m}$. (b) Section of a lymph node adjacent to that shown in (a). The outer cortex $(C)$ has been immunostained using monoclonal antibody OX12. The paracortex (P) and medullary cords (M) are shown. Bar: $200 \mu \mathrm{m}$.

approach to the problem was adopted - a quantitative assessment of the thymus-dependent areas on sections of the testicular lymph node and spleen.

The results indicate a significant increase in the volume of thymus-dependent cortex of the regional lymph nodes after unilateral vasectomy in comparison to sham operation. In contrast, there was no significant difference between vasectomized and sham-operated rats in the weights of the spleens or their content of thymus-dependent lymphoid tissue.

In this study, the spleen was examined at 3 months after vasectomy, the period with the most marked lymph node changes (McDonald \& Scothorne, 1986, 1989). The negative results of our analysis of the spleen, taken with those of Al-Saffar (1987), who assessed the quantity of white pulp in spleen sections from vasectomized and sham-operated rats from the same inbred colony as in the 
(a)

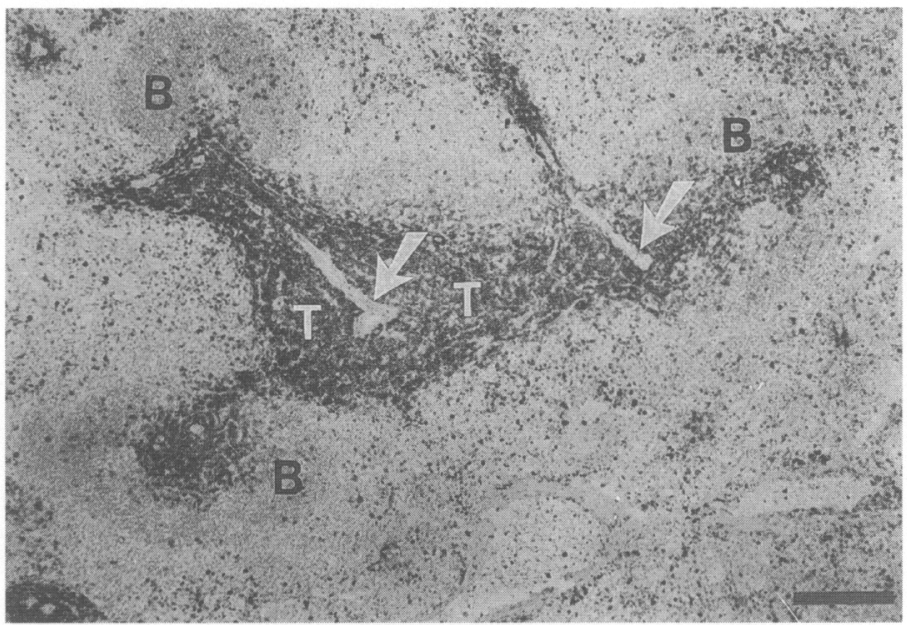

(b)

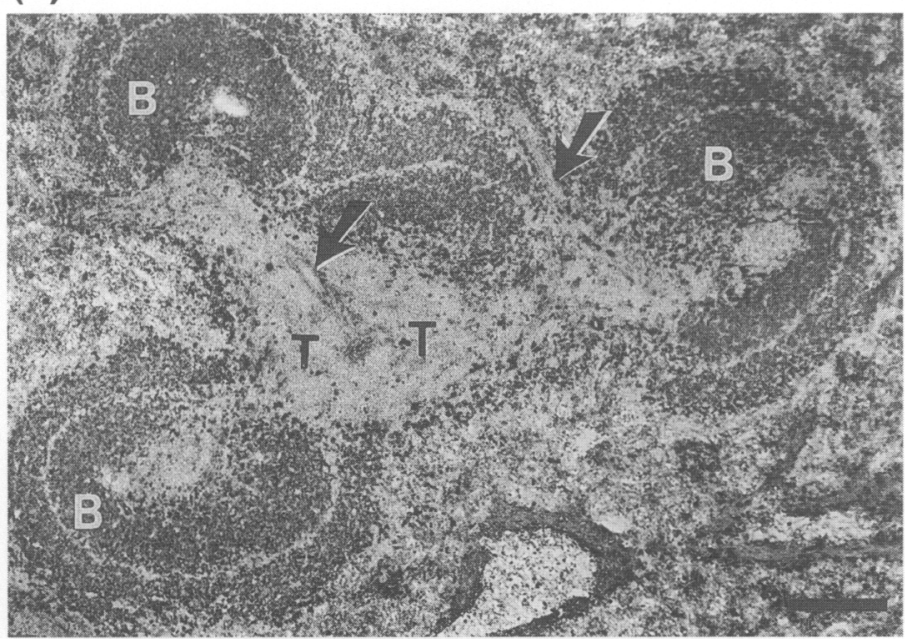

Fig. 3. (a) Section of spleen showing white pulp from a vasectomized rat. The T-cell areas (T) have been immunostained using monoclonal antibody W3/13. The unstained B-cell areas (B) are shown, as are profiles of the central arteriole (arrows). Bar: $200 \mu \mathrm{m}$. (b) Section of spleen showing white pulp adjacent to that shown in Fig. 3a. The B-cell areas (B) have been immunostained with monoclonal antibody OX12. The unstained T-cell areas (T) are shown, as are profiles of the central arteriole (arrows). Bar: $200 \mu \mathrm{m}$.

present study, suggest that the spleen in Swiss Albino rats does not exhibit morphological changes after vasectomy.

The findings indicate that, in rats, the immunological response to vasectomy involves both humoral and cell-mediated immunity. They also make clear that spermatozoan autoantigens are made available to the immune system by a lymphatic route and that the regional lymph nodes have an important role in mediating the immune response. In contrast, the bloodstream and spleen do not seem to be important in developing the responses.

The authors thank N. K. Bennett and M. Hughes for technical assistance. J. Halliday was supported by a medical student award from the Scottish Home and Health Department. 


\section{References}

Al-Saffar, R.A.S. (1987) Morphological Studies of the Immune Response to Vasectomy. PhD Thesis, University of Glasgow.

Bigazzi, P.E., Kosuda, L. \& Harnick, L. (1977) Sperm autoantibodies in vasectomised rats of different inbred strains. Science 197, 1282-1283.

Brannen, G.E., Kwart, A.M. \& Coffey, D.S. (1974) Immunologic implications of vasectomy. I. Cellmediated immunity. Fertility and Sterility 25, 508-514.

McDonald,S.W. \& Scothorne, R.J.(1986) On the response of the regional testicular lymph nodes after unilateral vasectomy in rats. Journal of Anatomy 144, 49-59.

McDonald, S.W. \& Scothorne, R.J. (1988) The lymphatic drainage of the epididymis and of the ductus deferens with reference to the immune response to vasectomy. Journal of Anatomy 158, 57-64.

McDonald, S.W. \& Scothorne, R.J. (1989) The response of the regional testicular lymph node six and nine months after vasectomy in rats. Journal of Anatomy 165, 251-257.

Nagarkatti, P.S. \& Rao, S.S. (1976) Cell-mediated immunity to homologous spermatozoa following vasectomy in the human male. Clinical and Experimental Immunology 26, 239-242.
Rumke, P. \& Titus, M. (1970) Spermagglutinin formation in male rats by subcutaneously injected syngeneic epididymal spermatozoa and by vasoligation or vasectomy. Journal of Reproduction and Fertility 21, 69-79.

Samuel, T., Kolk, A.H.J., Rumke, P. \& Van Lis, J.M.J. (1975) Autoimmunity to sperm antigens in vasectomised men. Clinical and Experimental Immunology 21, 65-74.

Shulman, S., Zappi, E., Ahmed, U. \& Davis, J.E. (1972) Immunologic consequences of vasectomy. Contraception 5, 269-278.

Tilney, N.L. (1971) Patterns of lymphatic drainage in the adult laboratory rat. Journal of Anatomy 109, 369-383.

Tumboh-Oeri, A.G. \& Roberts, T.K. (1979) Immunological and morphological consequences of vasectomy in the rabbit. Experientia 35, 675-676.

Van Noorden, S. (1986) Tissue preparation and immunostaining techniques for light microscopy. In Immunocytochemistry: Modern Methods and Applications 2nd edn, pp. 27-53. Eds J. M. Polak \& S. Van Noorden, Wright, Bristol.

Received 19 August 1991 